ESAIM: PROCEEDINGS AND SURVEYS, February 2015, Vol. 49, p. 91-101

Samira EL YACOUBI, Larbi AFIFI, El Hassan ZERRIK, Abdessamad TRIDANE, Editors

\title{
RISK ANALYSIS AND CONTROLLABILITY OF CREDIT MARKET
}

\author{
R. M. RAZAKANirina ${ }^{1}$ AND B. ChOpard ${ }^{2}$
}

\begin{abstract}
In this paper we present the behaviours of a simple credit market model built upon a static directed complex network topology. We found that the market structure plays a key role on the stability of such a model. Cyclic topologies accumulate more debt than acyclic ones, and when the total cash is less than the total debt, the market becomes unstable and exhibits chaotic behaviour. Exogenous creation of currency is used to control the market stability. Two regimes of control emerge depending on the credit market structure. First, power law control is needed for acyclic random topologies built upon Erdos-Rényi and Barabasi-Albert algorithms. Second, exponential control is needed to stabilize cyclic market built with the Watts-Strogatz algorithm.
\end{abstract}

Résumé. Dans cet article, nous présentons les comportements d'un modèle simplifié du marché du crédit. La structure du marché est construite autour d'un réseau complexe statique. Nos résultats montrent que la structure du marché joue un rôle crucial dans la stabilité du modèle. Les topologies cycliques accumulent plus de dettes que les topologies acycliques et lorsque la dette accumulée excède le montant total des liquidités, le marché devient instable et converge vers un comportement chaotique. La création exogène de liquidité est utilisée pour contrôler la stabilité du marché. Deux types de contrôle qui dépendent de la structure du marché du crédit émergent. Le premier type de contrôle est le contrôle suivant une loi de puissance. Ce contrôle est nécessaire pour stabiliser un marché basé sur les topologies acycliques du type réseaux de Erdos-Rényi et Barabasi-Albert. Le second type de contrôle est le contrôle suivant une loi exponentielle qui est nécessaire pour stabiliser un marché basé sur les topologies cycliques du type réseaux de Watts-Strogatz.

\section{INTRODUCTION}

Banking system can be viewed as a complex network [1-3] where nodes are banks and the links between nodes are the credit linkages between them. These links are highly volatile but can be aggregated and form emergent network structures. Knowing the characteristics of such networks, questions arise naturally about how to control such financial systems. This controllability aspect will give key tools to regulators, financial actors and policy makers to anticipate all necessary measures to avoid risk and systemic collapse of the whole system [4].

The controllability of complex network based on its structure gains in popularity since the seminal paper proposed by [5]. It gives new insights on how to control financial systems $[4,6]$. Nevertheless, it is important to specify the underlying dynamics behind each system. Recently, we have proposed abstract micro-economical models on complex networks to capture distinctive economical behaviours. First, we considered the dynamics of exchange of cash against goods in a complex network where links can be cut if trading is non profitable [7].

\footnotetext{
${ }^{1}$ Computer Science Department, University of Geneva; e-mail: ranaivo.razakanirina@unige.ch

2 Computer Science Department, University of Geneva; e-mail: bastien.chopard@unige.ch
} 
Second, we define a model in which nodes exchange salary against labour [8]. The labour is used to produce goods that can be sold on the cash-against-good market, thus coupling the two systems.

As a next step, we propose here a simple model of a banking system with a few homogeneous parameters and tackle the controllability related to its underlying dynamics. We will characterize the control applied to the model that drives the system to a desired state, especially insuring its stability.

The paper is organized as follows: first we describe the details of the credit market model. Second we analyse, in Section 2, the stability criteria of the model, and third we characterize, in Section 3, its controllability.

\section{Credit market model}

We consider a credit market consisting of a set $V$ of financial agents or banks connected according to a static interbank market structure. The balance sheet of each bank $i$ is very simple and takes in consideration the main two roles of bank: borrowing and lending. At a particular time iteration $t$, on the Asset side of the balance sheet, we have the total loan $\ell_{i}(t)$ granted to debtors and the cash reserve or liquidity $c_{i}(t)$. On the Liabilities side of the balance sheet, we have the initial capital $c_{i}(0)$ and the total debt $d_{i}(t)$ granted by creditors. At each time iteration $t$, the total of Assets is always equal to the total of Liabilities (equation (1)).

$$
c_{i}(t)+\ell_{i}(t)=c_{i}(0)+d_{i}(t) \quad \forall \text { bank } i .
$$

We notice that at $t=0$, the cash reserve is equal to the initial capital.

The dynamics within the credit market consists of two sequential operations. Both operations are made at each time iteration. First, each bank redeems mature debts to its creditors. Second, each bank either grants loans to its debtors with its remaining cash reserve or accepts debts from creditors or both.

The credit market model has three parameters that we assume to be identical for all agents:

- The level of investment $\alpha(0<\alpha<1)$, that defines the fraction of the cash reserve invested to make loan.

- The maturity date $\tau$ of the debt that defines the duration of a debt or in our case the number of time iteration before redeeming the debt. $\tau$ is a strictly positive integer.

- The interest rate $r$.

The homogeneity of these parameters across the system is not realistic but this simplification makes the study of their impacts simpler than with heterogeneous parameters. Regarding model complexity, our goal was to limit the number of model parameters. This limitation allows us to understand well the effects of each parameter on the emerging behaviours.

To illustrate how the balance sheet evolves, let us consider the following sequential operations processed by a bank A:

(1) Bank A is created with an initial capital of 10,000.-CHF. The level of investment $\alpha=0.6$. The balance sheet is

\begin{tabular}{l|l}
\multicolumn{2}{c}{ Assets } \\
\hline$c_{i}(t): 10,000$ & $c_{i}(0): 10,000$
\end{tabular}

(2) Bank A grants loan to debtors using the totality of its level of investment. Therefore we have the following balance sheet

\begin{tabular}{l|l}
\multicolumn{1}{c}{ Assets } & \multicolumn{1}{c}{ Liabilities } \\
\hline$c_{i}(t): 4,000$ & $c_{i}(0): 10,000$ \\
$\ell_{i}(t): 0.6 \times 10,000$ &
\end{tabular}

(3) Bank A accepts a total debt of 3,500.-CHF from creditors. The balance sheet becomes 


\begin{tabular}{l|l}
\multicolumn{1}{c}{ Assets } & \multicolumn{1}{c}{ Liabilities } \\
\hline$c_{i}(t): 4,000+3,500$ & $c_{i}(0): 10,000$ \\
$\ell_{i}(t): 6,000$ & $d_{i}(t): 3,500$
\end{tabular}

(4) Bank A redeems a debt of 2,500.-CHF to creditors. Thus

\begin{tabular}{l|l}
\multicolumn{1}{c}{ Assets } & \multicolumn{1}{c}{ Liabilities } \\
\hline$c_{i}(t): 7,500-2,500$ & $c_{i}(0): 10,000$ \\
$\ell_{i}(t): 6,000$ & $d_{i}(t): 3,500-2,500$
\end{tabular}

We can see from the above operations that the balance sheet expands during borrowing operation and contracts during redeeming operation. Lending operation has no effect on balance sheet size, defined as the total of assets or the total of liabilities.

The structure of static interbank market is a directed graph. Each graph node or vertex represents a bank. Each graph link or edge models the credit relationship between banks. The directed link $(i, j)$ between two banks $i$ and $j$ means that bank $i$ is the creditor of $j$ and bank $j$ is the debtor of $i$. We perform simulations on two categories of complex networks which are the Directed Acyclic Graphs (DAG) and Directed Cyclic Graphs (DCG). Both network categories are generated using random models.

We build a DAG using the random models of Erdos-Rényi and Barabasi-Albert. Let us first consider the Erdos-Rényi random model [9]. This model has two parameters which are the number of nodes $n$ and the connection probability $p$. In this model we start with $n$ nodes and link with probability $p$ a directed edge $(i, j)$ between any pair of distinct nodes $i$ and $j$. We choose low value of the probability $p$ to insure that the graphs generated by the Erdos-Rényi random model do not contain a cycle. In our case, a maximal connection probability of $p=0.25$ have been chosen. Furthermore, for each generated network, we test the existence of cycles and only networks without cycles are accepted as DAG.

Let us now consider the Barabasi-Albert model [10]. This model has three parameters which are the number of nodes $n$, number of initial nodes $m_{0}<n$ and the number of directed links $m$ that connect a new node to previously existing nodes. In this model, we start with $m_{0}$ nodes. The remaining nodes $n-m_{0}$ are added one by one. $m$ links connect each new node $i$ to the previously existing nodes $j$. The $m$ directed links are added with a probability $p_{j}$ which is proportional to the degree of the existing node $j$. The Barabasi-Albert model generates a scale-free directed graph which exhibits power-law degree distribution. This scaling phenomenon is mainly observed within real networks [11] and more particularly with interbank market structures $[3,12]$. In order to compare the connection probability of network generated by Barabasi-Albert and Erdos-Rényi models, we introduce the property $p_{\text {rand }}=M / n(n-1)$, where $M$ is the total number of edges. $p_{\text {rand }}$ is the equivalent random connection probability of the network generated by the Barabasi-Albert model. As with the ErdosRényi model, we test the existence of cycles of the networks generated by the Barabasi-Albert model and only networks without cycles are accepted as DAG.

The DCG are built using the random Watts-Strogatz model [13]. The existence of cycles is most likely with this model. The Watts-Strogatz model has three parameters which are the total number of nodes $n$, shortcut probability $\beta(0 \leq \beta \leq 1)$ and coordination number $k$ ( $k$ is an even number). In this model, we start with a $k$-regular ring graph (this graph type is a ring graph where each node has $k / 2$ incoming edges and $k / 2$ outgoing edges). Let us consider that the nodes are labelled from 0 to $n-1$. Each directed edge $(i, j)$, where $i=0, \ldots, n-1$ and $j=(i+1) \% n$, are rewired with a probability $\beta$ to a new directed edge $(i, q)$. Node $q$ is selected randomly among the existing nodes. A duplicate edge is not permitted. The equivalent random connection probability is $p_{\text {rand }}=M / n(n-1)$, where $M$ is the total number of edges of the network generated by the Watts-Strogatz model. The existence of cycle is always tested and only networks with cycles are accepted as DCG.

Lending-borrowing dynamics are subject to risk. We consider that creditors are risk averse creditors and debtors are risk neutral debtors. It means on the one side that loans are granted with minimal risk to the 
TABLE 1. Balance sheet of a bank A at time $t$ in case of liquidity shortage.

\begin{tabular}{l|l}
\multicolumn{1}{c}{ Assets } & \multicolumn{1}{c}{ Liabilities } \\
\hline$c_{i}(t): 500$ & $c_{i}(0): 10,000$ \\
$\ell_{i}(t): 10,500$ & $d_{i}(t): 1,000$
\end{tabular}

TABLE 2. Parameters of the simulated networks.

\begin{tabular}{cccccccc}
\hline Name & Type & $n$ & $M$ & $p$ & $m_{0} / m$ & $\beta / k$ & $p_{\text {rand }}$ \\
\hline RND & DAG (Erdos-Rényi) & 50 & 637 & 0.25 & - & - & - \\
RND1 & DAG (Erdos-Rényi) & 200 & 485 & 0.012 & - & - & - \\
RND2 & DAG (Erdos-Rényi) & 200 & 585 & 0.015 & - & - & - \\
RND3 & DAG (Erdos-Rényi) & 200 & 10,071 & 0.25 & - & - & - \\
SF1 & DAG (Barabasi-Albert) & 200 & 235 & - & $10 / 1$ & - & 0.0059 \\
SF2 & DAG (Barabasi-Albert) & 200 & 775 & - & $30 / 2$ & - & 0.0195 \\
SF3 & DAG (Barabasi-Albert) & 200 & 1,675 & - & $50 / 3$ & - & 0.0420 \\
WS & DCG (Watts-Strogatz) & 200 & 1,000 & - & - & $0.01 / 10$ & 0.0251 \\
\hline
\end{tabular}

creditor. The cash reserve level is the risk indicator. Therefore, a loan is granted proportionally to the level of liquidity (cash reserve) owned by debtors. On the other side, the debts are always accepted by debtors. This fact mimics the bank-depositor relationships.

\section{Stability REgion}

The risk in banking system is the shortage of liquidity creating financial distress within credit market. In the presented model, we identify two sources of liquidity shortage. The first one is the lack of cash reserve to redeem debts. To illustrate this case, let us consider the balance sheet of a bank $\mathrm{A}$ at time iteration $t$ (Table 1). From this balance sheet, as $c_{i}(t)<d_{i}(t)$, bank A becomes illiquid if the total debt of 1,000.-CHF should be redeemed at $t$. This situation is mainly due to the time lag between redeeming to creditors and redeeming from debtors operations that we will analyse later according to the investment $\alpha$ and the loan duration $\tau$.

The second source of liquidity shortage is the interest rate $r$. The system is a closed system and the total cash reserve does not change over time. When interest rate is not null, the nominal amount to redeem at maturity date (consisting of due interest rate and the principal amount) is higher than the principal amount. Therefore, introducing interest rate forces the system to find more cash than existing initially within the credit market.

In order to highlight why the liquidity shortage arises, we focus first on the evolution of the total debt as a function of the three parameters of the model. We define the total debt $d_{t o t}(t)$ as the sum of the debts of all banks at time $t$

$$
d_{t o t}(t)=\sum_{\forall i \in V} d_{i}(t)
$$

We also introduce the total cash reserve $c_{t o t}$. This quantity is constant over time as the system is closed as long as there is no injection or deletion of cash. This quantity is defined at a particular time iteration $t$ as

$$
c_{t o t}(t)=\sum_{\forall i \in V} c_{i}(t)
$$

We perform simulations on DAG (networks generated with Erdos-Rényi and Barabasi-Albert random models) and DCG (networks generated with Watts-Strogatz random model). Table 2 summarizes the generated random networks presented in this work with their parameters.

Each bank holds initially the same amount of capital. We assume however that $c_{t o t}(0)=5,000,000$. Setting $c_{t o t}(0)$ to the same value for each simulation allows us to compare between them the simulation results. As the simulated topologies are generated by random models, the total number of banks does not play a key role on the 


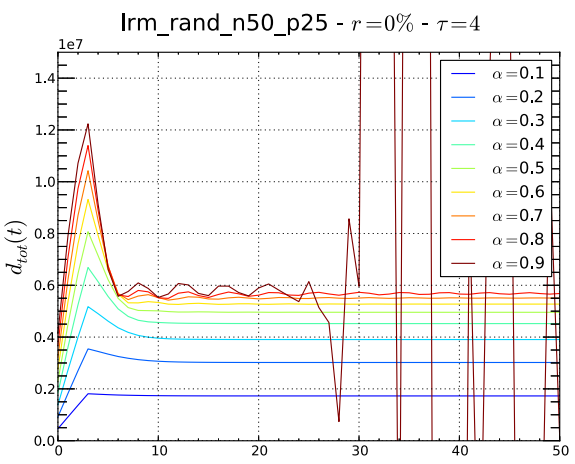

(a) With $\alpha \in[0.1,0.9], \tau=4$ and $r=0 \%$.

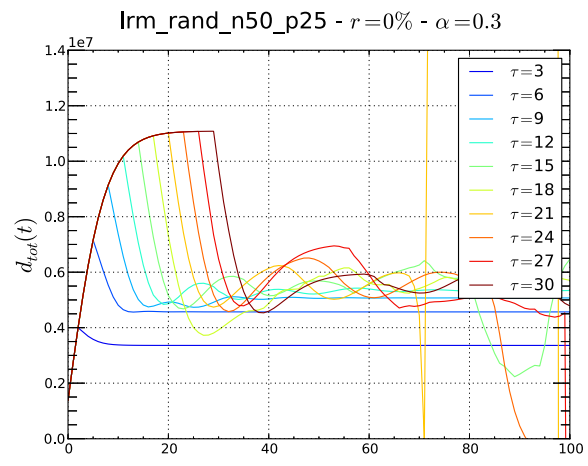

(b) With $\alpha=0.3, \tau \in[3,30]$ and $r=0 \%$.

Figure 1. Total debt $d_{t o t}(t)$ versus time $t$ for the RND DAG topology with 50 banks and connection probability $p=0.25$. $c_{t o t}(0)=5 \times 10^{6}$. It is most likely to have unstable state when $d_{t o t}(t)>c_{t o t}(t)$. (a) The system converges to steady state for $\alpha \in[0.1,0.6]$. Oscillations start to appear for $\alpha \in[0.7,0.8]$. Chaotic state is observed for $\alpha=0.9$ starting from $t=25$. (b) The system converges to steady state for $\tau \in[3,6]$. Unstable states are observed from $\tau=9$.

characteristics of the generated network. The numbers of banks we chose in this work are $n=50$ and $n=200$ as these numbers best represent the obtained results. During the simulations, we vary the homogeneous values of the level of investment $\alpha$ (from 0.1 to 0.9 ), loan duration $\tau$ (from 1 to 30 ) and interest rate $r$ (from $0 \%$ to $4 \%$ ).

Figure 1 depicts the time evolution of the total debt for our DAG. We found that steady state exists after the convergence to a stable level of the total debt. This behaviour is observed for instance when $\alpha=0.2, \tau=4$ and $r=0 \%$ (see Figure 1(a)), or when $\alpha=0.3, \tau=3$ and $r=0 \%$ (see Figure 1(b)). A steady state is also observed for our DCG topology (see Figure 2). For the considered DCG, the total debt becomes stable for instance when $\alpha=0.2, \tau=4$ and $r=0 \%$ (see Figure 2(a)), or when $\alpha=0.6, \tau=3$ and $r=0 \%$.

It is interesting to see for both the DAG and DCG topologies that the total debt level becomes chaotic with high values of $\alpha$ and $\tau$. On the other hand, low values of $\alpha$ and $\tau$ stabilise the total debt level.

From the steady state and chaotic observations on DAG and DCG, we introduce the definition of steady state frontier. This frontier defines the limit values of the level of investment $\alpha$ and the loan duration $\tau$ that separate the steady state behaviour from the chaotic behaviour. In other words, this frontier delineates the investment and the loan duration values avoiding liquidity shortage and insuring the stability of the credit market. Figure 3 depicts the steady state frontiers for the Erdos-Rényi DAG and Watts-Strogatz DCG respectively considered in Figure 1 and Figure 2. In both cases we used here $r=0 \%$.

We found that the steady state frontier follows a power law and can be expressed as follows

$$
\alpha(\tau)=a \tau^{-\gamma},
$$

where the constant $a$ and the exponent $\gamma$ depends on the considered credit market. For the Erdos-Rényi DAG presented in Figure 3(a), we have $a=0.75$ and $\gamma=0.95$. For the Watts-Strogatz DCG presented in Figure 3(b), we have $a=0.41$ and $\gamma=1.05$. These estimated values of $a$ and exponent $\gamma$ should be treated with caution as it is given using visual estimation.

These results confirm that the stability of credit market is strongly related to the level of investment and the loan duration. Short-term debt with high investment and long-term debt with low investment are most likely the cases that insure financial stability.

More particularly, the evolution of total debt with $r=4 \%, \alpha=0.2$ and $\tau=6$ for Erdos-Rényi DAG is depicted in Figure 4(b). We can see that instead of remaining stable from $t \in[10,100]$ as with $r=0 \%$ (Figure $4(\mathrm{a}))$, the total debt level begins to rise from $t=10$ until reaching a completely chaotic state. These results 


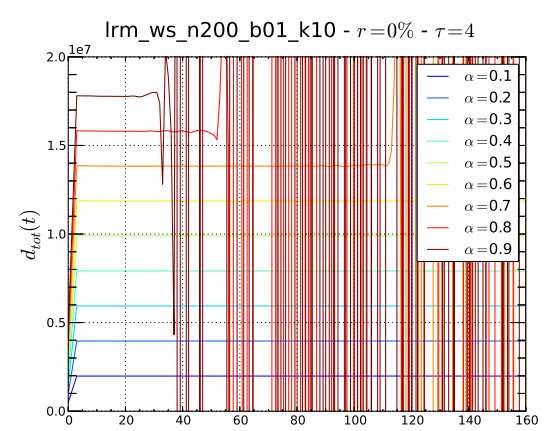

(a) With $\alpha \in[0.1,0.9], \tau=4$ and $r=0 \%$.

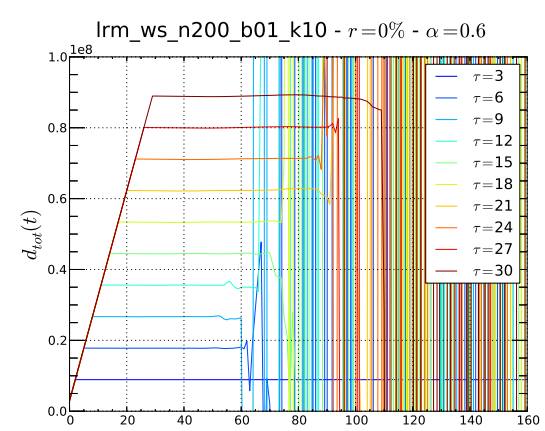

(b) With $\alpha=0.6, \tau \in[3,30]$ and $r=0 \%$.

Figure 2. Total debt $d_{\text {tot }}(t)$ versus time $t$ for the WS DCG topology with 200 banks, a coordination number $k=10$ and a short-cut probability $\beta=0.01$. $c_{\text {tot }}(0)=5 \times 10^{6}$. (a) For the considered time interval $(t \in[0,160])$, the system converges to steady state for $\alpha \in[0.1,0.6]$. Instabilities start when $\alpha \in[0.7,0.9]$. Notice that the system converges to steady state even $d_{\text {tot }}(t)$ is greater than $c_{\text {tot }}$. (b) For the considered time interval, only the simulations with $\tau=3$ leads to steady state.

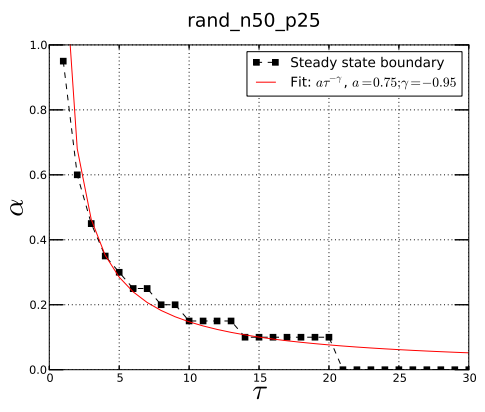

(a)

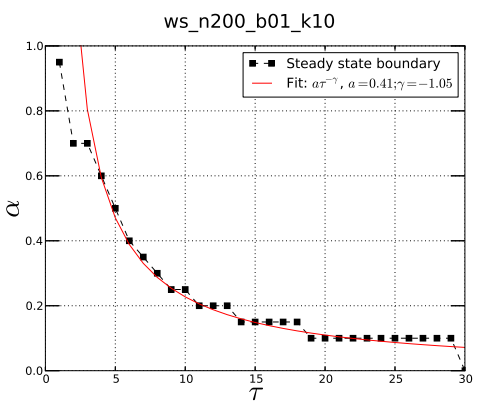

(b)

FiguRE 3. Steady state frontiers for (a) Erdos-Rényi DAG (RND) and (b) Watts-Strogatz DCG (WS). The red line is the estimated power law fit. All combination of $\alpha$ and $\tau$ below the red line correspond to the stability region.

confirm our initial assumption on the effect of interest rate on a closed system. The total debt increases due to loan interests. We found that the system is always unstable when the interest rate is not equal to zero. In that case, the total cash reserve does not rise while the total debt rises and needs more cash to be redeemed.

Further analysis of the total debt level in comparison with the total cash reserve demonstrate that chaotic behaviours are most likely observed when the total cash reserve $c_{t o t}(t)$ is less than the total debt level $d_{t o t}(t)$. We found the steady state condition expressed as follows

$$
\begin{cases}\text { steady state, } & \text { if } d_{t o t}(t)<c_{t o t}(t), \forall t \\ \text { most likely chaotic, } & \text { otherwise } .\end{cases}
$$




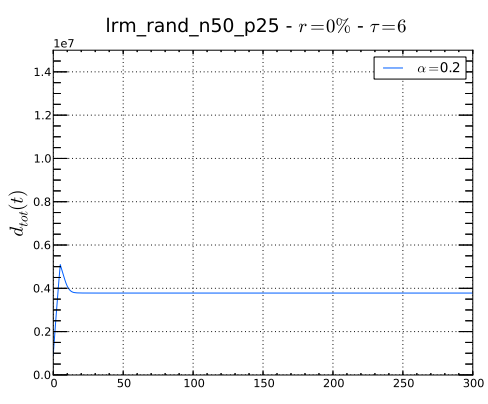

(a) $r=0 \%$

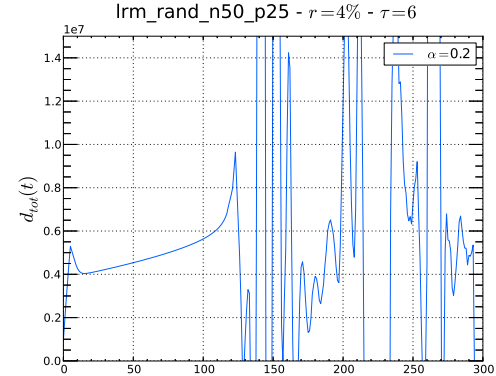

(b) $r=4 \%$

Figure 4. The steady state (see (a)) observed with $\alpha=0.2$ and $\tau=6$ for Erdos-Rényi DAG (RND) becomes chaotic (see (b)) from $t=130$ when introducing interest rate.

\section{Controllability: Driver nodes And COST}

We have seen in the previous section that when the cash reserve is insufficient to redeem debt, the system goes to chaotic state. This is particularly the case when the interest rate of granted loans is not null. To address liquidity shortage due to the interest rate, we inject new cash so that each bank has the necessary cash reserve to continue to work. This cash injection corresponds to the intervention of Central Bank (CB) as a lender of last resort when bank liquidity is insufficient.

Let us consider the balance sheet of a bank A depicted in Table 1. In order to avoid liquidity shortage, bank A should borrow 500.-CHF from CB to redeem its total debt of 1,000.-CHF. We consider that cash injection from $\mathrm{CB}$ corresponds to money creation, expressed using the balance sheet depicted in Table 3. CB creates money $\left(u_{i}(t)\right)$ and lends the created money to bank A. This operation expands the CB balance sheet.

TABLE 3. Balance sheet of Central Bank (CB) to support bank A (see Table 1).

\begin{tabular}{c|l}
\multicolumn{2}{c}{ Assets } \\
\hline$\ell_{i}(t): 500$ & $u_{i}(t): 500$
\end{tabular}

Bank A expands also its balance sheet with additional debt from CB $\left(u_{i}(t)\right)$ and increases its cash reserve. The new financial situation of bank $\mathrm{A}$ is given by Table 4 .

TABLE 4. New balance sheet of bank A after CB intervention.

\begin{tabular}{l|l}
\multicolumn{1}{c}{ Assets } & \multicolumn{1}{c}{ Liabilities } \\
\hline$c_{i}(t): 500+500$ & $c_{i}(0): 10,000$ \\
$\ell_{i}(t): 10,500$ & $d_{i}(t): 1,000$ \\
& $u_{i}(t): 500$
\end{tabular}

The credit market model is a non-linear dynamical system. The CB intervention can be seen as a control applied on such dynamical system to avoid liquidity shortage. We characterize this control by two time dependent values. The first value is the number of driver nodes $n_{D}(t)$. A driver node is a bank within the credit market model that is at least supported one time by CB. In other words, this node is among the fragile nodes of the market. The second value is the total amount of cash $u_{t o t}(t)$ injected to support the whole credit market and to insure its stability. $u_{t o t}(t)$ is the total cost of control. This value is defined at each time iteration $t$ by 


$$
u_{\text {tot }}(t)=\sum_{\forall i \in V} u_{i}(t) .
$$

Figures 5 and 6 depict the characteristics of controls applied respectively to the DAG and DCG topologies when the interest rate $r=0 \%$. It is interesting to see that two regimes of controls emerge from the simulations. First, Erdos-Rényi and Barabasi-Albert DAG exhibit periodic power law control (see Figure 5) with a period $\tau$. We observe different peaks of control, each peak exhibiting power law cash injection decay. The cost of control can be expressed for one peak as

$$
u_{\text {tot }}(t)=a t^{-\gamma} \text {, where } t=k \tau \text { with } k \in \mathbb{Z}^{+} .
$$

The control periodicity is due to the fact that the redeeming step is synchronized among the agents, due to the choice of an homogeneous loan duration.

For an equivalent random connection probability $p_{\text {rand }}$, we found that the total debt is higher for the BarabasiAlbert DAG than for the Erdos-Rényi DAG. This situation implies that more cash is necessary to stabilize a market with the Barabasi-Albert DAG topology compared to that necessary to stabilize the Erdos-Rényi DAG. We notice for both topologies that the number of driver nodes increases when the connection probability increases.

Second, Watts-Strogatz DCG topology needs exponential control (see Figure 6). By contrast with previous DAG topologies, the control is applied constantly. The cost of control can be expressed as

$$
u_{t o t}(t)=a e^{b t}
$$

This result can be understood using the evolution of the total debt level. Figure 6 shows that the level of debt from $t \in[20,40]$ is already higher than $c_{t o t}$. This situation increases the cash injection. The cyclic topology accumulates more debts than acyclic topology. Therefore when the total cash increases, the total level of debt increases faster than the total cash and then drives the cash injection to higher value. The cycle will continue, thus explaining the exponential control exhibited by cyclic market topologies. We also observe that the number of driver nodes tends asymptotically to a fixed value. For the considered topology, we have $n_{D}(t) \rightarrow 30 \%$ of the total number of nodes when $t$ goes to infinity. This result indicates that cyclic topology exhibits cyclic controlled node.

Figure 7 depicts the control needed to stabilize Erdos-Rényi DAG, Barabasi-Albert DAG and Watts-Strogatz DCG topologies with a positive interest rate. The total level of debt diverges for all the considered topologies. The controls are chaotic but exponential trends are observed both for the number of driver nodes and for the cost to control.

\section{Conclusion}

We presented a simple credit market model consisting of a set of banks that interact between them using static credit linkages. The dynamics of each bank consists of two simple sequential steps: redeeming mature debts to creditors and lending to debtors. The loans are granted with homogeneous loan duration and homogeneous interest rate. An homogeneous level of investment defines the amount to lend to debtors.

We found that steady states may exist, i.e. that the credit market is stable and the total debt level is under control, provided that level of investment and loan duration are properly chosen. We found that the frontier of the $\alpha-\tau$ stability region follows a power law. Beyond this steady state frontier, the system becomes chaotic. In that case, the total debt is greater than the total cash. The existence of cycles within the credit linkages topology amplifies the level of total debt and drives the market to an unstable state. We notice that the market is always unstable with positive interest rate.

Cash injection is used to control and to drive the system to a stable state. We found on the one hand that for Erdos-Rényi and Barabasi-Albert DAG topologies, the control is periodic and follows a series of decaying power laws. The period is equal to the loan duration. On the other hand, an exponential control is necessary to stabilize Watts-Strogatz DCG topologies. The credit market with positive interest rate exhibits also exponential 


$$
n=200-r=0 \%-\tau=21-\alpha=0.4
$$
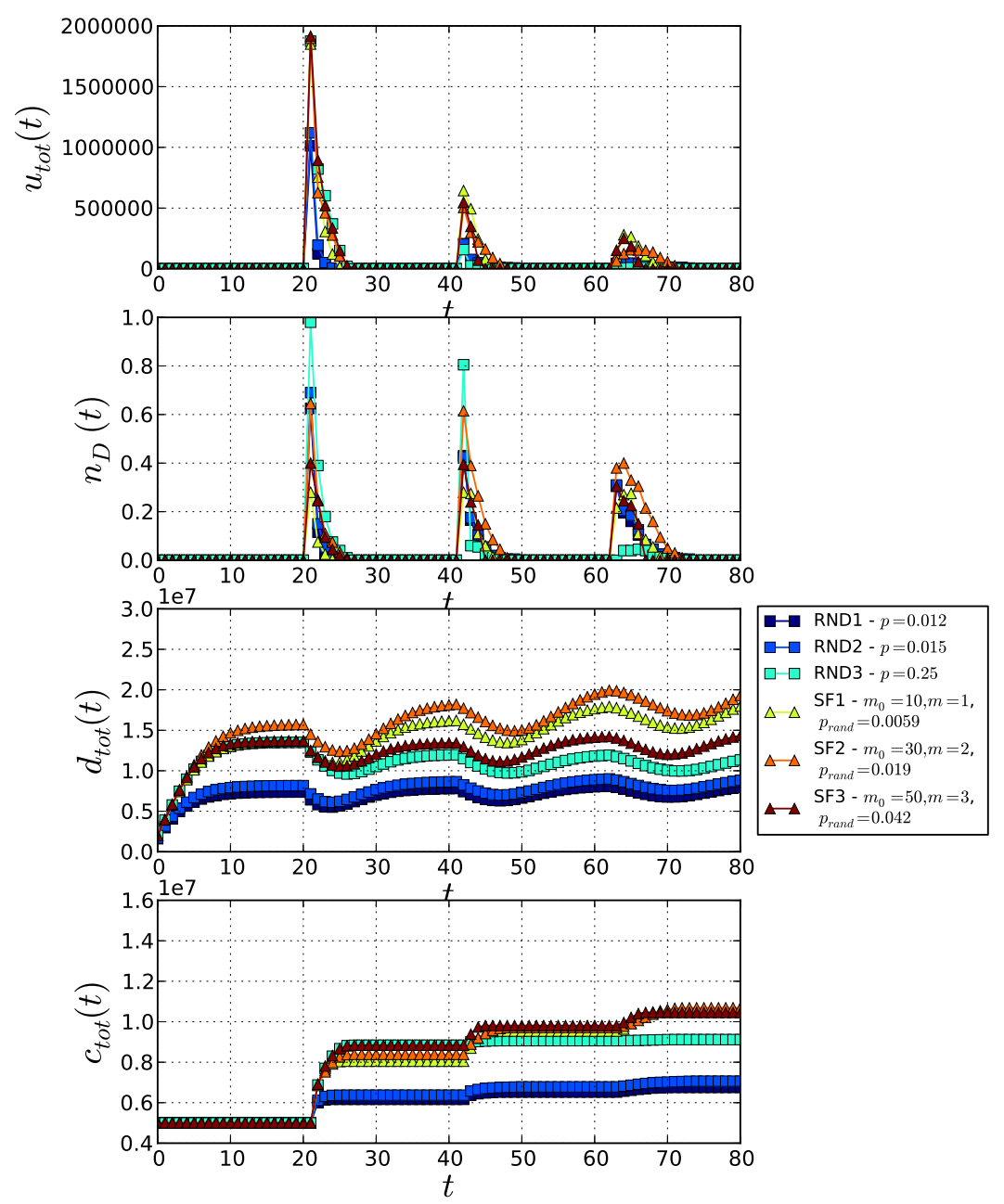

FiguRE 5. Control characterization for Erdos-Rényi and Barabasi-Albert DAG topologies with $r=0 \%$. Periodic power law cash injection is observed in the top figure forming multiple periodic peaks. Each peak corresponds to power law cash injection. The same behaviour is observed for driver nodes (second figure from top).

control trend. We can argue that such a case corresponds, in real market, to an uncontrollable situation. Indeed, Central Bank cannot inject permanently an exponentially growing amount of cash to stabilize the system.

The authors acknowledge the financial support from the Swiss National Science Foundation and the University of Geneva. 
ws_n200_b01_k10 - $r=0 \%-\alpha=0.6$
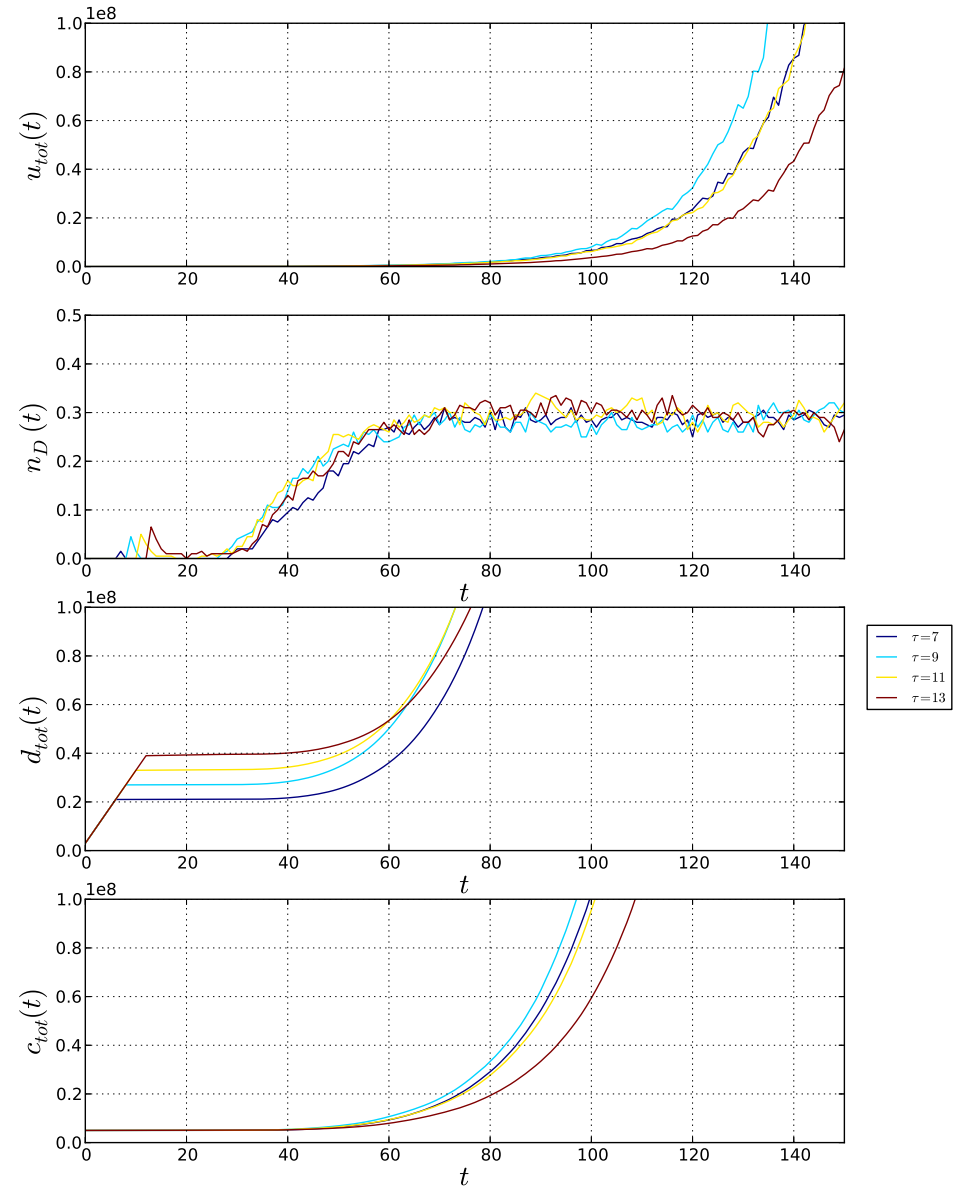

Figure 6. Control characterization for Watts-Strogatz DCG topology (WS) with $r=0 \%$. Exponential control is observed with an asymptotic number of driver nodes.

\section{REFERENCES}

[1] M. Raddant, "Structure in the italian overnight loan market," Journal of International Money and Finance, vol. 41, pp. 197213, Mar. 2014.

[2] D. Garlaschelli, S. Battiston, M. Castri, V. D. P. Servedio, and G. Caldarelli, "The scale-free topology of market investments," Physica A: Statistical Mechanics and its Applications, vol. 350, pp. 491-499, May 2005.

[3] M. Boss, H. Elsinger, M. Summer, and S. Thurner 4, "Network topology of the interbank market," Quantitative Finance, vol. 4 , no. 6 , pp. $677-684,2004$.

[4] D. Delpini, S. Battiston, M. Riccaboni, G. Gabbi, F. Pammolli, and G. Caldarelli, "Evolution of controllability in interbank networks," Nature Scientific Report, vol. 3, Apr. 2013.

[5] Y.-Y. Liua, J.-J. Slotine, and B. Albert-László, "Controllability of complex networks," Nature, vol. 473, p. 167-173, May 2011.

[6] S. Thurner and S. Poledna, "DebtRank-transparency: Controlling systemic risk in financial networks," Nature - Scientific Report, May 2013. 


$$
n=200-r=4 \%-\tau=5-\alpha=0.2
$$
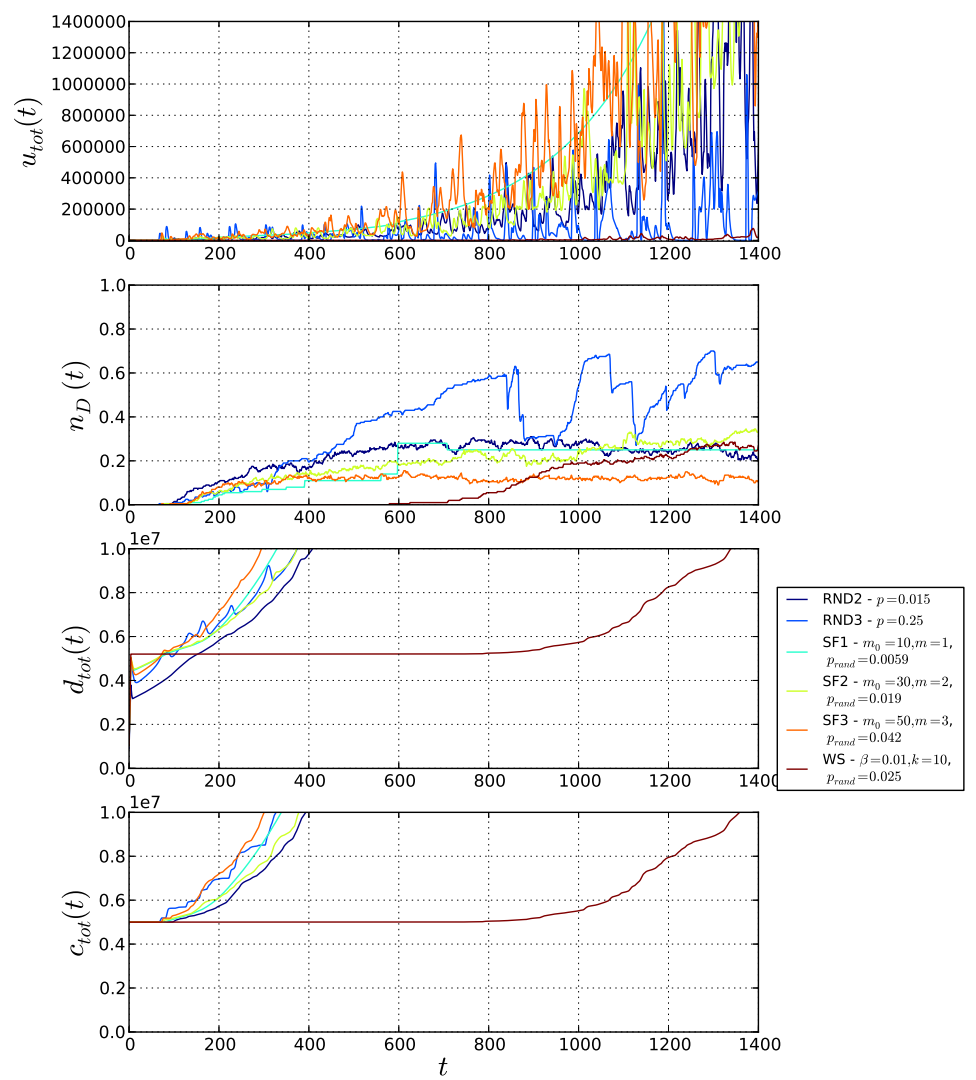

FIGURE 7. Control characterization when the interest rate $r>0 \%$ (here $r=4 \%$ ) with complex networks consisting of 200 banks. Chaotic control is observed and the general trend is an exponential control (top figure).

[7] R. M. Razakanirina and B. Chopard, "Using cellular automata on a graph to model the exchanges of cash and goods," in Cellular Automata, p. 163-172, Springer, 2010.

[8] R. M. Razakanirina and B. Chopard, "Labour and goods market dynamics using an abstract microeconomical model," Acta Phys Pol B, Proc Suppl, vol. 5, no. 1, pp. 131-143, 2012.

[9] P. Erdös and A. Rényi, "On random graphs, i," Publicationes Mathematicae (Debrecen), vol. 6, pp. 290-297, 1959.

[10] R. Albert and A.-L. Barabási, "Statistical mechanics of complex networks," Reviews of modern physics, vol. 74, no. 1, p. 47, 2002.

[11] E. Estrada, The Structure of Complex Networks: Theory and Applications. New York, NY, USA: Oxford University Press, Inc., 2011.

[12] G. De Masi, G. Iori, and G. Caldarelli, "Fitness model for the italian interbank money market," Physical review. E, Statistical, nonlinear, and soft matter physics, vol. 74, p. 066112, Dec. 2006.

[13] D. J. Watts and S. H. Strogatz, "Collective dynamics of 'small-world' networks," Nature, vol. 393, pp. 440-442, June 1998. 